\begin{tabular}{|c|l|}
\hline Title & Large wave vector phonons in highly dispersive crystal s: Phonon-focusing effects \\
\hline Author(s) & Tamura, Shin-ichiro \\
\hline Citation & $\begin{array}{l}\text { Physical Review B, 28(2), 897-909 } \\
\text { https://doi.org/10.1103/PhysRevB.28.897 }\end{array}$ \\
\hline Issue Date & 1983-07-15 \\
\hline Doc URL & http://hdl.handle.net/2115/5955 \\
\hline Rights & Copyright $\odot$ 1983 A merican Physical Society \\
\hline Type & article \\
\hline File Information & PRB28-2.pdf \\
\hline
\end{tabular}

Instructions for use 


\title{
Large-wave-vector phonons in highly dispersive crystals: Phonon-focusing effects
}

\author{
Shin-ichiro Tamura \\ Department of Engineering Science, Hokkaido University, Sapporo 060, Japan \\ (Received 7 March 1983)
}

\begin{abstract}
The anisotropic propagation or the focusing of large-wave-vector acoustic phonons in highly dispersive crystalline $\mathrm{Ge}$ is studied in detail. Our analysis is entirely based on an elaborate Born-von Kármán model of the lattice dynamics which is constructed so as to reproduce the observed phonon dispersion curves very accurately. Effects of the lattice dispersion upon the phonon focusing are found to be discernible above $0.3 \mathrm{THz}$ and become drastic at frequencies higher than 1 THz. These observations are made by studying complementarily the frequency dependences of the following objects: the shape of constant-frequency surfaces, the locations of phonon caustics, the distributions of phonons in real space, the angular dependences of phonon intensity, and the structure of group-velocity surfaces. A brief discussion is also given of the effects of isotopes which act to damp significantly the ballistic phonon intensity at frequencies in the $1-\mathrm{THz}$ range.
\end{abstract}

\section{INTRODUCTION}

In recent years there has been considerable activity in the area of high-frequency phonon propagation in solids. $^{1-7}$ This has been supported by the development of the techniques for generating and detecting acoustic phonons of frequencies up to several THz. ${ }^{8}$ Some important information to be gained by the experiments utilizing such high-frequency nonequilibrium phonons may be the effects of lattice dispersion upon the phonon transport in the thermal-frequency range. A few years ago, Ulbrich et al. found in high-purity GaAs that near-zone-edge transverse-acoustic (TA) phonons propagate in ballistic fashion over macroscopic distances and reveal several features characteristic of a dispersive medium. ${ }^{1}$ Subsequently, Dietsche et al. observed in Ge the ballistic flux patterns of the TA phonons of frequencies higher than 0.7 $\mathrm{THz}$, which are remarkably different from those obtained for lower-frequency phonons. ${ }^{3}$ The large feature of the findings by Dietsche et al. is now believed to be interpretable, ${ }^{9,10}$ based on the idea of phonon focusing ${ }^{11,12}$ modified by acoustic dispersion.

The possible detection of the high-frequency TA phonons at low temperatures is currently understood in terms of their lifetime due to anharmonic phonon-phonon interactions. The most important three-phonon processes in the regime $\omega=2 \pi v \gg k_{B} T / \hbar$ are shown to yield a short lifetime for longitudinal-acoustic (LA) phonons in proportion to $\omega^{-5}$, but a very long one for the TA phonons as described by the factor $\exp \left(a \hbar \omega / k_{B} T\right)$, where $a$ is a constant of the order of unity. ${ }^{13}$ The former prediction on the LA phonons has recently been established experimentally by Baumgartner et al. for a weakly dispersive and quasiisotropic $\mathrm{CaF}_{2}$ sample. $^{5}$ It should be noted here that natural $\mathrm{Ge}$ and $\mathrm{GaAs}$ include isotopes. The scattering of the phonons by isotopic atoms is highly frequency dependent $^{14}$ and severely restricts the ballistic phonon transport at $\mathrm{THz}$ frequencies. Indeed, owing to the presence of this scattering mechanism by the isotopes, Dietsche et al. had to prepare thin Ge samples of $0.5-\mathrm{mm}$ thickness to observe sharp images of ballistic phonons higher than $0.7 \mathrm{THz}$ (which are detected by $\mathrm{Pb}$-oxide- $\mathrm{Pb}$ tunneling junction detector). ${ }^{3}$

By a recent theoretical work on the isotope scattering of dispersive phonons in $\mathrm{Ge},{ }^{15}$ the scattering rate at frequencies near $1 \mathrm{THz}$ is shown to grow more rapidly than $\omega^{4}$, the frequency dependence being valid in the low-frequency limit. According to the results, the mean free path of the phonons at $1.5 \mathrm{THz}$ is reduced to $10 \mu \mathrm{m}$ or less. Hence in order to detect the unscattered phonons higher than 1 $\mathrm{THz}$, thin-film samples of thickness down to several micrometers are required in principle. When experiments that observe the ballistic transport of the phonons up to several $\mathrm{THz}$ become feasible, their anisotropic spatial distributions due to the phonon focusing are expected to be quite different from those of the low-frequency phonons by the effects of the dispersion.

The purpose of the present work is to make a comprehensive study of anisotropic phonon conduction, or phonon focusing, at dispersive frequencies. To do this, we should first understand correctly the dynamics of lattice vibrations of the medium beyond the continuum approximation. In this paper we shall devote our attentions to highly dispersive crystalline $\mathrm{Ge}$, for which the nature of dispersion relations has been well understood by experiments ${ }^{16,17}$ with slow neutrons. The key entity which plays a fundamental role throughout our investigations is the so-called dynamical matrix of the lattice. The knowledge on the various properties of the phonons, such as the frequency, the phase and group velocities, and the curvature of the constant-frequency surface ( $\omega$-surface) of the phonons, is derived straightforwardly from the eigenvalues or by appropriate differentiations of the relevant dynamical matrix. Hence as a start we try to construct the dynamical matrix which reproduces very accurately the data for the phonon dispersion curves being obtained by the inelastic neutron scatterings. We make this in the framework of the lattice dynamics according to the Born-von Kármán scheme, ${ }^{18}$ which will be described in the next section. The three-dimensional representations of the $\omega$ surfaces at typical frequencies in the $1-\mathrm{THz}$ range are then exhibited for TA mode phonons. The implications of the shapes of 
these $\omega$ surfaces to the phonon focusing near zoneboundary frequencies will also be remarked on in Sec. II.

The traditional description of the phonon focusing is based on a ray picture of the phonons. In this geometrical representation for the phonons, there exist certain directions called caustics along which the phonon flux becomes singular. ${ }^{19}$ The caustics are associated with folding edges in the multivalued ray, or group-velocity surfaces, and provide important insight into the nature of the focusing. The sharp amplifications of the phonon intensity have actually been observed by experiments in the predicted caustic directions. ${ }^{20,21}$ A further global understanding of the focusing is gained by plotting the distribution of the phonons in the real space when the phonons are assumed to be distributed uniformly over the $\omega$ surface. In Sec. III the frequency dependences of such configurations as the phonon-enhancement map and the location of the caustics will be studied.

A more quantitative understanding of the focusing will be provided by calculating the enhancement factor of the phonons introduced originally by Maris $^{12}$ for lowfrequency phonons. The enhancement factor measures an enhancement of phonon flux in a specified direction relative to the magnitude it would have in an isotropic medium, and thereby is connected directly to the phonon intensity. In Sec. IV we shall study the angular dependence of the phonon intensity which is substantially determined by the enhancement factor. The structures found in the directional properties of the phonon intensity will also be discussed with reference to the group-velocity surfaces.

Owing to the presence of highly frequency-dependent scattering by the isotopes, it will be rather hard, in practice, to arrange for the phonons of $\mathrm{THz}$ frequencies an experimental situation in which the mean free path of the phonons may be much longer than the distance between the phonon source and the detector. In the experiments by Dietsche et al. ${ }^{3}$ the path lengths of the phonons are comparable to, or shorter than, their mean free path. In these cases, the production of diffusive phonons which yield predominant background signals should act to interrupt the observation of anisotropic, ballistic flux patterns due to the phonon focusing. We shall briefly describe in Sec. $\mathrm{V}$ the effects of the isotope scattering upon the propagation of near 1-THz phonons.

Throughout this work we shall concentrate our analyses mainly upon the TA phonons, and the LA phonons will be touched upon only briefly. This is because the focusing of the LA phonons is rather moderate up to the frequency of about $3 \mathrm{THz}$ and does not give rise to any sharp feature in the phonon intensity to be observed vividly. ${ }^{9}$ In addition, the strong anharmonic interaction and isotope scattering act to prevent severely the detection of the otherwise observable sharp focusing patterns of high-frequency nearzone-boundary LA phonons.

\section{LATTICE DYNAMICS}

Here we briefly describe the lattice dynamics of Ge we have employed. In the harmonic approximation the properties of the lattice vibrations in a crystal, or of the phonons throughout the Brillouin zone, are deduced on the basis of the equations

$$
\begin{aligned}
& \sum_{\beta, \sigma^{\prime}}\left[\omega^{2} \delta_{\alpha \beta} \delta_{\sigma \sigma^{\prime}}-G_{\alpha \beta}\left(\sigma, \sigma^{\prime} \mid \overrightarrow{\mathrm{q}}\right)\right] e_{\beta}\left(\sigma^{\prime}\right)=0 \\
&(\alpha=1,2,3 ; \sigma=1,2, \ldots, r),
\end{aligned}
$$

where $\sigma$ and $\sigma^{\prime}$ specify $r$ atoms which consist of a unit cell and $\vec{e}$ stands for the polarization vector of the lattice which is normalized according to $|\overrightarrow{\mathrm{e}}|=1$. The $3 r \times 3 r$ Hermitian matrix $\underline{G}$ is called a dynamical matrix which can be expressed in terms of interatomic force constants $\Phi$ 's as ${ }^{22}$

$$
\begin{aligned}
G_{\alpha \beta}\left(\sigma, \sigma^{\prime} \mid \overrightarrow{\mathrm{q}}\right)= & \left(m_{\sigma} m_{\sigma^{\prime}}\right)^{-1 / 2} \\
& \times \sum_{l^{\prime}} \Phi_{\alpha \beta}\left(l, \sigma ; l^{\prime}, \sigma^{\prime}\right) e^{-i \overrightarrow{\mathrm{q}} \cdot\left[\overrightarrow{\mathrm{x}}(l)-\overrightarrow{\mathrm{x}}\left(l^{\prime}\right)\right]},
\end{aligned}
$$

where $m_{\sigma}$ is the atomic mass and $\vec{x}(l)$ is the position vector of the $l$ th unit cell. In the Born-von Kármán model of the lattice dynamics, the interatomic force constants are regarded as adjustable parameters whose values are to be determined from a comparison of the predictions of the theory with experimental data.

With respect to developing quantitative discussions of the phonon focusing at $\mathrm{THz}$ frequencies, we need to know correct dispersive characters of the phonons in Ge. In the present work we have employed the Born-von Kármán scheme and then tried to construct a dynamical matrix which may reproduce as accurately as possible the data of the dispersion obtained by the inelastic neutron scatterings, ${ }^{16,17}$ as well as the data of elastic constants. ${ }^{23}$ In this formal force-constant model, by adding interactions extending to the atoms at a distance, we can obtain, in principle, the dynamical matrix which may yield the phonon dispersion relations fitted to any degree of accuracy to those measured by the experiments, provided that the crystal is intrinsically regarded to be harmonic. In fact, Herman showed a long time ago that the interatomic forces at least up to fifth-nearest neighbors are required to reproduce qualitatively the notable flattening of the dispersion curves in the TA branches of $\mathrm{Ge}^{24}$ More recently, Zdetsis and Wong extended this scheme including the interatomic force constants up to eighth neighbors. ${ }^{25}$ Their results fit considerably the dispersion curves along the principal $\Delta-, \Sigma$-, and $\Lambda$-symmetry directions. However, two force constants in the seventh neighbor have been overlooked.

In the present work we have tried to search the bestfitted force-constant parameters also up to eighth-nearest neighbors. The values of these 31 force constants we have found are given in Table I with the notations of Herman. ${ }^{24}$ The searching procedure of the parameters we adopted is the same as that in Ref. 25; that is, the experimental data used in fitting process are 70 evenly distributed points on the phonon dispersion curves along three principal directions in addition to the Raman frequency and three elastic constants. With the use of the force constants in Table I, we have diagonalized the dynamical matrix and found

$$
\left\langle\left|\frac{\Delta v}{v}\right|\right\rangle_{\mathrm{av}}=\frac{1}{n} \sum_{i=1}^{n} \frac{\left|v_{i}^{\mathrm{fit}}-v_{i}^{\text {expt }}\right|}{v_{i}^{\text {expt }}}=0.74 \%,
$$

where $n=71$ is the total number of fitted frequencies. The 
TABLE I. Fitted force constants for $\mathrm{Ge}\left(10^{4} \mathrm{dyn} / \mathrm{cm}\right)$.

\begin{tabular}{lrlr}
\hline \hline$\alpha^{(1)}$ & 4.2533 & $\mu^{(6)}$ & -0.0084 \\
$\beta^{(1)}$ & 3.7007 & $\lambda^{(6)}$ & 0.0670 \\
& & $v^{(6)}$ & -0.0945 \\
$\mu^{(2)}$ & 0.3317 & $\delta_{+}{ }^{(6)}$ & 0.0359 \\
$\lambda^{(2)}$ & -0.7210 & $\delta_{-}^{(6)}$ & 0.0539 \\
$v^{(2)}$ & 0.2019 & & \\
$\delta^{(2)}$ & 0.1686 & $\alpha^{(7)}$ & -0.0591 \\
& & $\beta^{(7)}$ & 0.1068 \\
$\mu^{(3)}$ & -0.0696 & $\mu^{(7)}$ & 0.1560 \\
$\lambda^{(3)}$ & -0.2547 & $\lambda^{(7)}$ & -0.0209 \\
$v^{(3)}$ & -0.2035 & $\nu^{(7)}$ & 0.1128 \\
$\delta^{(3)}$ & -0.1377 & $\delta^{(7)}$ & -0.0262 \\
& & & 0.0161 \\
$\mu^{(4)}$ & 0.1699 & $\mu^{(8)}$ & -0.1565 \\
$\lambda^{(4)}$ & -0.2556 & $\lambda^{(8)}$ & 0.0133 \\
$\mu^{(5)}$ & & $v^{(8)}$ & -0.0490 \\
$\lambda^{(5)}$ & 0.1484 & $\delta^{(8)}$ & \\
$v^{(5)}$ & 0.5472 & & \\
$\delta^{(5)}$ & 0.1057 & & \\
\hline \hline
\end{tabular}

value of Eq. (3) is in accordance with the estimated uncertainties of the measured phonon frequencies ranging from $0.3 \%-0.5 \%$ for optical branches and $0.3 \%-1 \%$ for acoustic branches. ${ }^{16}$

The comparison of the calculated frequencies with the experimental ones is shown in Fig. 1. The coincidence of our calculations with the experiments is quite satisfactory except for the LA phonons on the Brillouin-zone boundaries $L-K$. This close coincidence tells us that the effects of isotopes and anharmonicity upon phonon frequency are rather small in Ge. The slight deviations recognized for the LA phonons at the zone boundaries may be remedied by adding interactions of atoms beyond the eighth neighbors, or by including the anharmonic effects. ${ }^{26}$

In Fig. 2 we have displayed the computer plot of the one-phonon density of states together with the contribution of each branch. ${ }^{27}$ Comparing it with the results for the density of states by Nelin and Nilsson ${ }^{17}$ who employed the extended sampling method with experimental data, our results are much more smooth and exhibit numerous critical points more distinctly.

Plotted in Figs. 3(a)-3(c) are the sections by the (110) plane of the $\omega$ surfaces in the wave-vector space which are obtained for three acoustic branches. (We refer to the phonons in the lower and higher TA branches as T1 and T2 phonons, respectively.) In these maps we immediately recognize conspicuous deformations of the $\omega$ surfaces at dispersive frequencies. Since the focusing properties of the phonon are closely connected with the shape of the $\omega$ surface through the definition of the group-velocity vector, it is such that the deformations of the $\omega$ surfaces should lead to drastic alterations of the focusing behaviors of the large-wave-vector phonons. For instance, the strong magnification of the $\mathrm{T} 2$ phonon focusing in the [110] axis and also in the direction rotated about $25^{\circ}$ away from the [001] axis are expected at near-zone-boundary frequencies. In addition, the sharp focusings of the LA phonons in the [001] as well as [111] directions are expected at frequencies much higher than $1 \mathrm{THz}$ in spite of the fact that the former direction is the defocusing one in the low-frequency limit. These situations for the LA phonons have already been described in Ref. 9.

Here we remark that the correct understanding of the

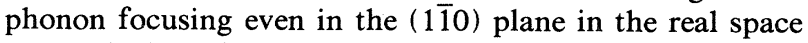
spanned by the group-velocity vector requires the knowledge on the whole three-dimensional shapes of the $\omega$ surfaces. This is because there exists, in general, more than one direction of the wave vectors for which the group velocities of the phonons point in the same direction, though they may be different in magnitude. In Figs. $4(a)-4(d)$ we have illustrated the first octants of the $\omega$ surfaces for the TA phonons at 0.3 and $1.5 \mathrm{THz}$. In these figures the regions of both the negative curvature (saddle areas) and positive curvature (concave and convex areas) are indicated explicitly. (The determination of the curvature of the $\omega$ surface will be described in Sec. III.) Now it should be noted that the former frequency can be viewed as a typical one in the weak dispersive region because the shapes of the $\omega$ surfaces at this frequency are nearly identical to those in the low-frequency limit. ${ }^{28,29}$ In contrast, the latter one may be regarded as the frequency in the highly dispersive region because of marked deformations of the $\omega$ surfaces.

The effects of the acoustic dispersion upon the shape of
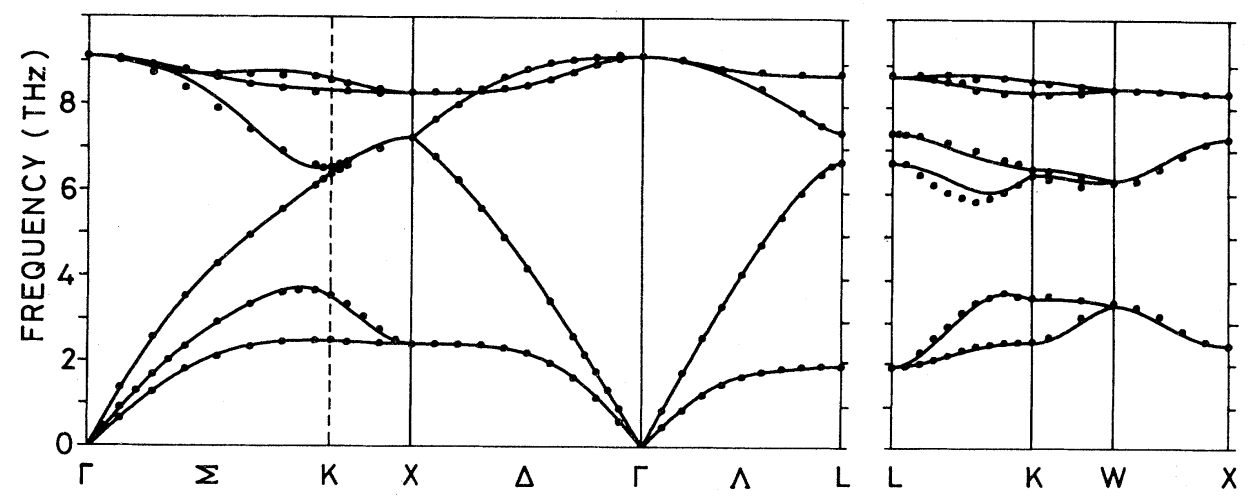

FIG. 1. Phonon dispersion curves for Ge. Solid lines show our calculations. Experimental values (closed circles) are from Refs. 16 and 17. 


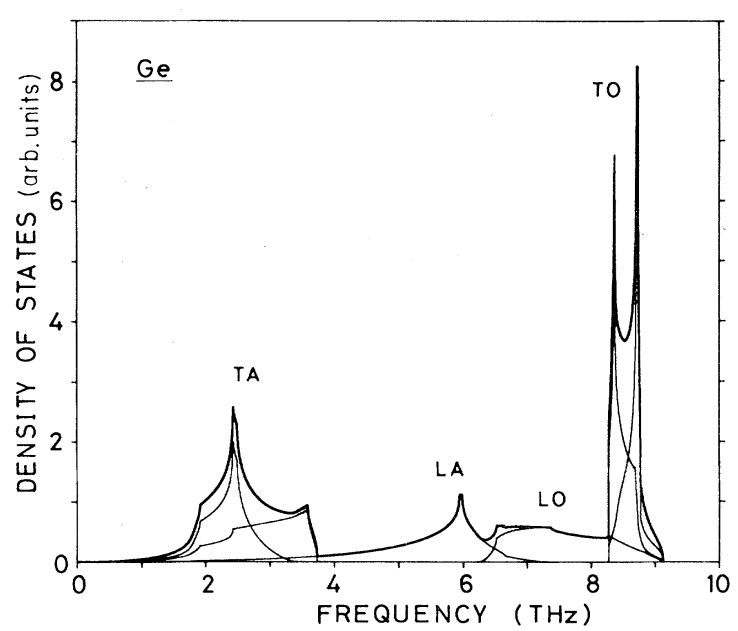

FIG. 2. Computer plot of one-phonon density of states calculated for Ge.

the $\omega$ surfaces are qualitatively understood by making reference to Fig. 4. For the T1 mode, by increasing the frequency the saddle areas which extend from the threefold [111] direction toward the [100] direction become narrow and the fourfold concave areas which are confined to exist in the proximity of the [100] direction shrink gradually. The latter areas are checked to disappear at a frequency close to $1.2 \mathrm{THz}$. On the other hand, for the T2 mode, the saddle areas which spread on either side of the (100) plane broaden by increasing the frequency. These changes in the $\omega$ surfaces make us conceive the reduction (magnification) of the T1 (T2) phonon focusing in the directions existing in the vicinity of the (110) [(100)] plane. However, for much more detailed understanding of the focusing characteristics beyond those described hitherto we need to examine the directional properties of the phonons in the real space. This requires extensive numerical calculations based on the force-constant model described in this section and will be carried out in the following sections.

\section{CAUSTICS AND DISTRIBUTIONS OF PHONONS IN THE REAL SPACE}

Owing to the presence of crystal anisotropy, the $\omega$ surface of the phonons consists, in general, of areas of both the positive and negative curvatures which are separated from each other by the zero-curvature parabolic points. The direction of the group velocity is, by its definition, given by the outward normal of the $\omega$ surface. According$1 y$, an infinity of the wave vectors is mapped into a single direction in the real space which is parallel to the surface normal at the parabolic point. In other words, in such a direction, which is called a caustic, the phonon flux explodes. Thus the caustic provides a singularity in the phonon intensity which characterizes the phonon focusing. In this section we shall investigate the effects of the lattice dispersion upon the locations of the caustic and also upon the phonon distributions in the real space.
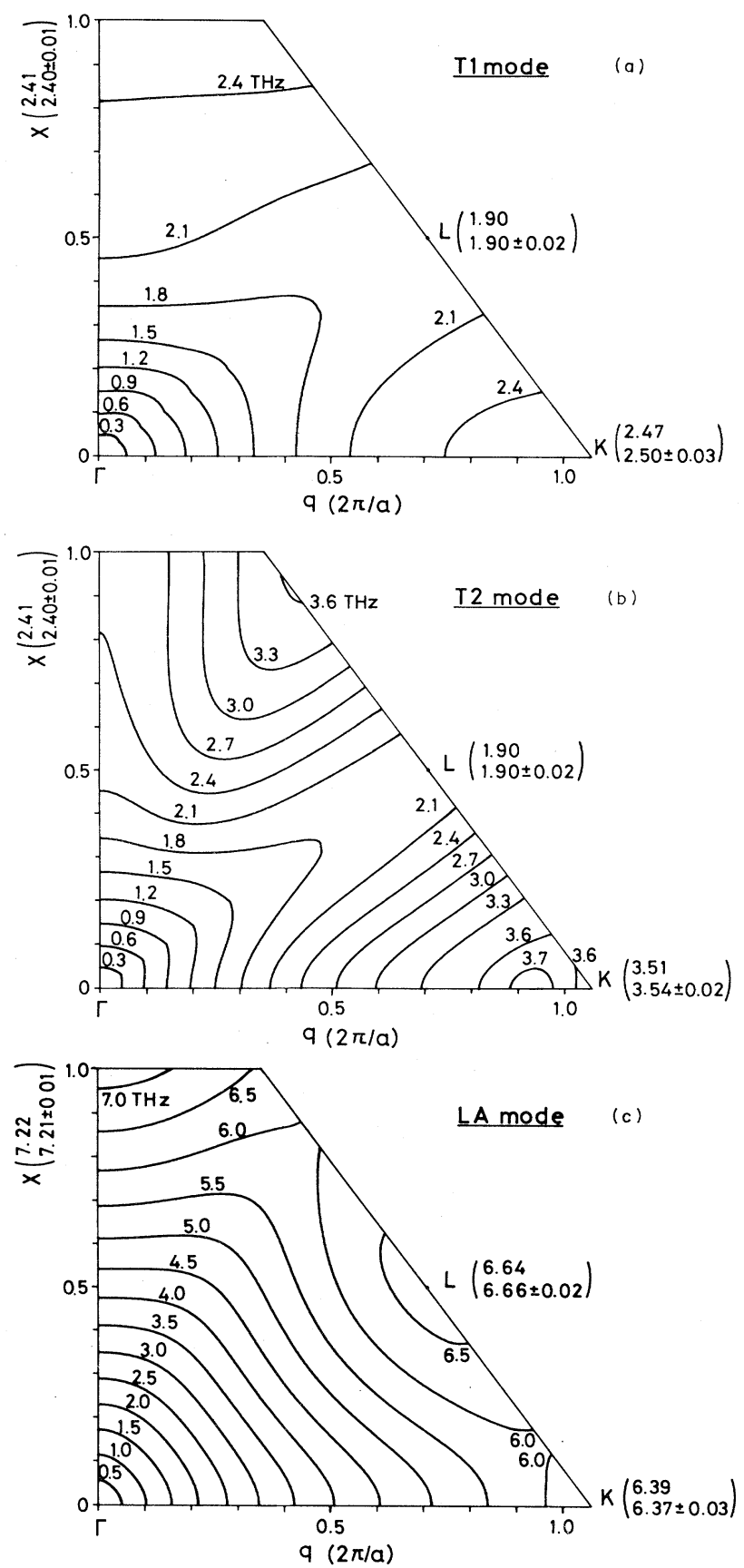

FIG. 3. Sections by (110) plane of calculated $\omega$ surfaces of (a) T1 mode, (b) T2 mode, and (c) LA mode. (Frequencies indicated are in units of THz.) Frequencies at zone-boundary points $X, L$, and $K$ are shown in parentheses; the calculated value is in the upper row and the experimental value is in the lower row.

\section{A. Caustics}

To begin, let us denote by $d \Omega(\overrightarrow{\mathrm{q}}, j)$ an infinitesimal solid angle occupied by $j$-mode phonons in the wave-vector space, and by $d \Omega(\overrightarrow{\mathrm{v}}(\overrightarrow{\mathrm{q}}, j))$ the corresponding solid angle in the real space spanned by the group-velocity vector $\vec{v}$. 

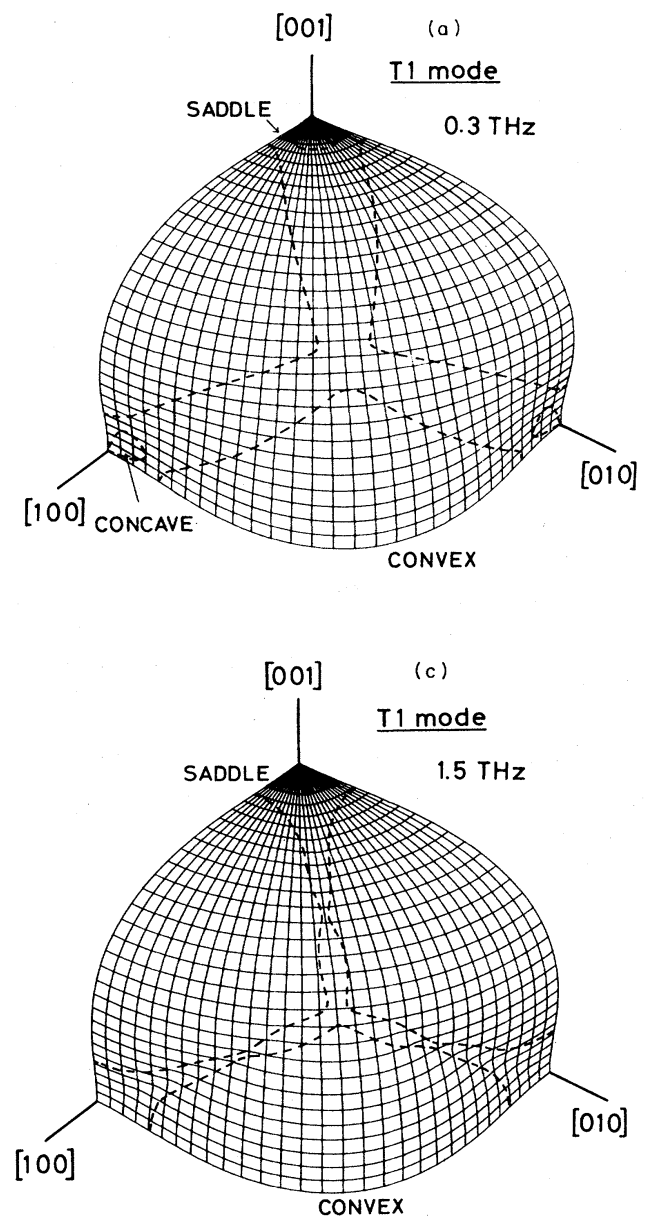
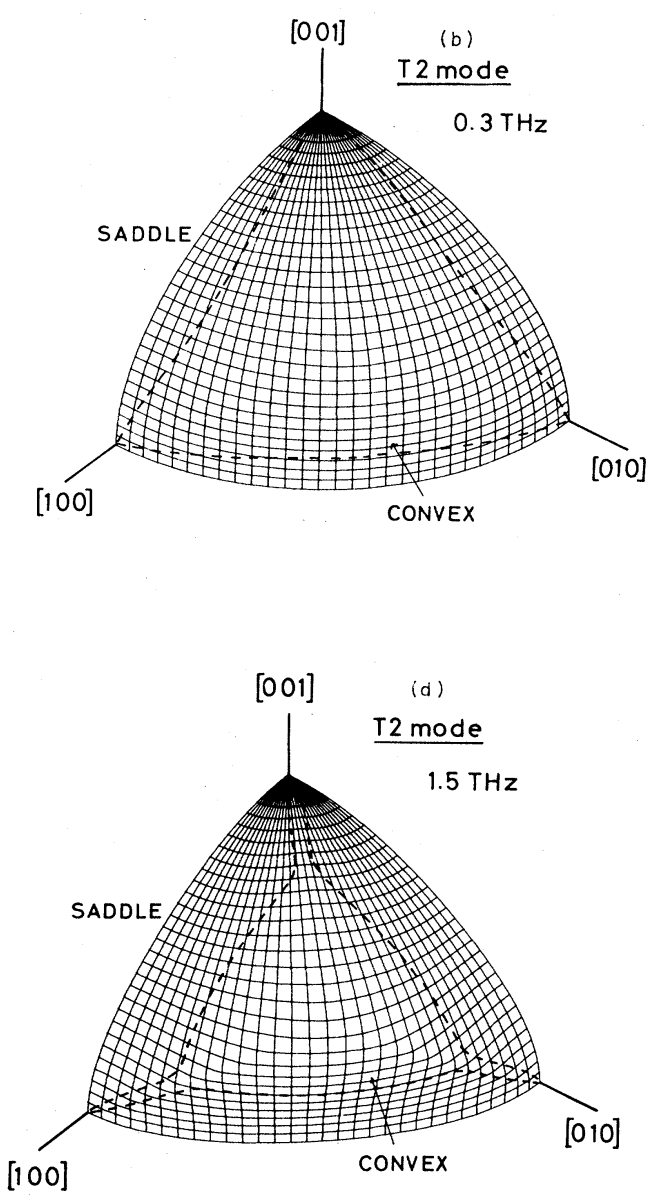

FIG. 4. Plots for the first octants of the $\omega$ surfaces of (a) $\mathrm{T} 1$ mode at $0.3 \mathrm{THz}$, (b) T2 mode at $0.3 \mathrm{THz}$, (c) T1 mode at $1.5 \mathrm{THz}$, and (d) T2 mode at $1.5 \mathrm{THz}$. The dashed lines which separate the saddle regions from the concave or convex regions are the traces of the parabolic points with vanishing curvature.

Then it can be deduced that the Gaussian curvature $K_{\omega}$ of the $\omega$ surface [defined by $\omega=\omega(\vec{q}, j)$ ] is related to these solid angles as ${ }^{30}$

$$
\frac{q^{2} K_{\omega}(\overrightarrow{\mathrm{q}}, j)}{\cos \theta(\overrightarrow{\mathrm{q}}, j)}=\frac{d \Omega(\overrightarrow{\mathrm{v}}(\overrightarrow{\mathrm{q}}, j))}{d \Omega(\overrightarrow{\mathrm{q}}, j)}=A^{-1}(\overrightarrow{\mathrm{q}}, j),
$$

where $q=|\overrightarrow{\mathrm{q}}|$ and $\theta$ is the angle between $\overrightarrow{\mathrm{q}}$ and $\overrightarrow{\mathrm{v}}$. It should be pointed out that the first equality of Eq. (4) holds irrespective of the presence of the dispersion, and the second equality defines the phonon-enhancement factor $A$. The ratio of the solid angles, or the enhancement factor $A$, is calculated by the formula, ${ }^{12}$

$$
\begin{aligned}
A^{-1}(\overrightarrow{\mathrm{q}}, j)= & {\left[\frac{q}{v(\overrightarrow{\mathrm{q}}, j)}\right]^{3} } \\
& \times \frac{\overrightarrow{\mathrm{v}}(\overrightarrow{\mathrm{q}}, j) \cdot\left[d \overrightarrow{\mathrm{v}}_{1}(\overrightarrow{\mathrm{q}}, j) \times d \overrightarrow{\mathrm{v}}_{2}(\overrightarrow{\mathrm{q}}, j)\right]}{\overrightarrow{\mathrm{q}} \cdot\left(d \overrightarrow{\mathrm{q}}_{1} \times d \overrightarrow{\mathrm{q}}_{2}\right)},
\end{aligned}
$$

where $v=|\overrightarrow{\mathrm{v}}|, d \overrightarrow{\mathrm{q}}_{1}$ and $d \overrightarrow{\mathrm{q}}_{2}$ are infinitesimal wave vec- tors (noncollinear to each other), and $d \vec{v}_{1}$ and $d \vec{v}_{2}$ are the corresponding increments of the group-velocity vector associated with the changes $\overrightarrow{\mathrm{q}} \rightarrow \overrightarrow{\mathrm{q}}+d \overrightarrow{\mathrm{q}}_{1}$ and $\overrightarrow{\mathrm{q}} \rightarrow \overrightarrow{\mathrm{q}}+d \overrightarrow{\mathrm{q}}_{2}$, respectively. Here, in order that $A$ given by Eq. (5) describes through Eq. (4) correctly the curvature of the $\omega$ surface at dispersive frequencies, the vectors $d \overrightarrow{\mathrm{q}}_{1}$ and $d \overrightarrow{\mathrm{q}}_{2}$ should be taken to be tangential to the surface. Of course, this restriction can be lifted in the low-frequency limit because the shape of the $\omega$ surface is independent of the magnitude of the wave vector. However, it is essential to obtain the correct enhancement factor and the curvature of the $\omega$ surface at a fixed and finite frequency.

The calculation of $A$ in accordance with Eq. (5) requires the knowledge of the derivatives of the frequency with respect to the wave vector up to second order, i.e., $\partial \omega / \partial q_{\alpha}=v_{\alpha}$ and $\partial^{2} \omega / \partial q_{\alpha} \partial q_{\beta}=\partial v_{\alpha} / \partial q_{\beta}$. In terms of the dynamical matrix, these can be derived from the following formulas:

$$
\frac{\partial \omega^{2}(\overrightarrow{\mathrm{q}}, j)}{\partial q_{\alpha}}=C_{\alpha}^{(1)}(\overrightarrow{\mathrm{q}} ; j, j),
$$




$$
\begin{aligned}
\frac{\partial^{2} \omega^{2}(\overrightarrow{\mathrm{q}}, j)}{\partial q_{\alpha} \partial q_{\beta}}= & C_{\alpha \beta}^{(2)}(\overrightarrow{\mathrm{q}} ; j, j) \\
& +\sum_{j^{\prime}(\neq j)}\left(\frac{C_{\alpha}^{(1)}\left(\overrightarrow{\mathrm{q}} ; j, j^{\prime}\right) C_{\beta}^{(1)}\left(\overrightarrow{\mathrm{q}} ; j^{\prime}, j\right)}{\omega^{2}(\overrightarrow{\mathrm{q}}, j)-\omega^{2}\left(\overrightarrow{\mathrm{q}}, j^{\prime}\right)}+\text { c.c. }\right),
\end{aligned}
$$

where

$$
\begin{aligned}
C_{\mu 1, \ldots, \mu n}^{(n)}\left(\overrightarrow{\mathrm{q}} ; j, j^{\prime}\right)= & C_{\mu 1, \ldots, \mu n}^{(n)} *\left(\overrightarrow{\mathrm{q}} ; j^{\prime}, j\right) \\
=\sum_{\alpha, \beta} \sum_{\sigma, \sigma^{\prime}} e_{\alpha}^{*}(\sigma \mid \overrightarrow{\mathrm{q}}, j) & \\
& \times \frac{\partial^{(n)} G_{\alpha \beta}\left(\sigma, \sigma^{\prime} \mid \overrightarrow{\mathrm{q}}\right)}{\partial q_{\mu 1} \cdots \partial q_{\mu n}} \\
& \times e_{\beta}\left(\sigma^{\prime} \mid \overrightarrow{\mathrm{q}}, j^{\prime}\right)
\end{aligned}
$$

and the summation over $j^{\prime}$ extends over the optical as well as acoustic branches. We have obtained the curvature and then the caustics of the TA phonons at the dispersive frequencies by deriving analytical expressions for both the first and second derivatives of the dynamical matrix, which includes the interatomic forces up to eighth neighbors.

The locations of calculated caustics of the TA phonons are shown in Figs. 5(a)-5(d) for 0.3, 0.7, 1.0, and $1.5 \mathrm{THz}$, respectively. The diagrams are the polar projections of the irreducible $\frac{1}{48}$ th sector of the section of the caustic surfaces by unit sphere in the real space. The effects of the acoustic dispersion upon the locus of the T2 phonon caustics are rather simple, that is, with increasing the frequency the caustics shift rapidly away from the (100) plane. This behavior has been supported by experiments. ${ }^{3}$ The theoretical frequency dependence of the locus of T2 caustics is given in Fig. 6. According to this figure, the experimental value of the opening angle of the $T 2$ caustics obtained with the tunneling junction detector corresponds to the frequency of $0.8 \mathrm{THz}$.

In contrast, the change of the singularity patterns for the $\mathrm{T} 1$ phonons is more complex. We see that there are two sets of the caustics near the [100] directions. Firstly, the inner structure of the caustics in the proximity of the [100] axes contracts with increasing the frequency and then disappears at a frequency close to $1.2 \mathrm{THz}$ (see Fig. 6). (This structure of the caustics is originated from the fourfold closed curves of the parabolic points encircling
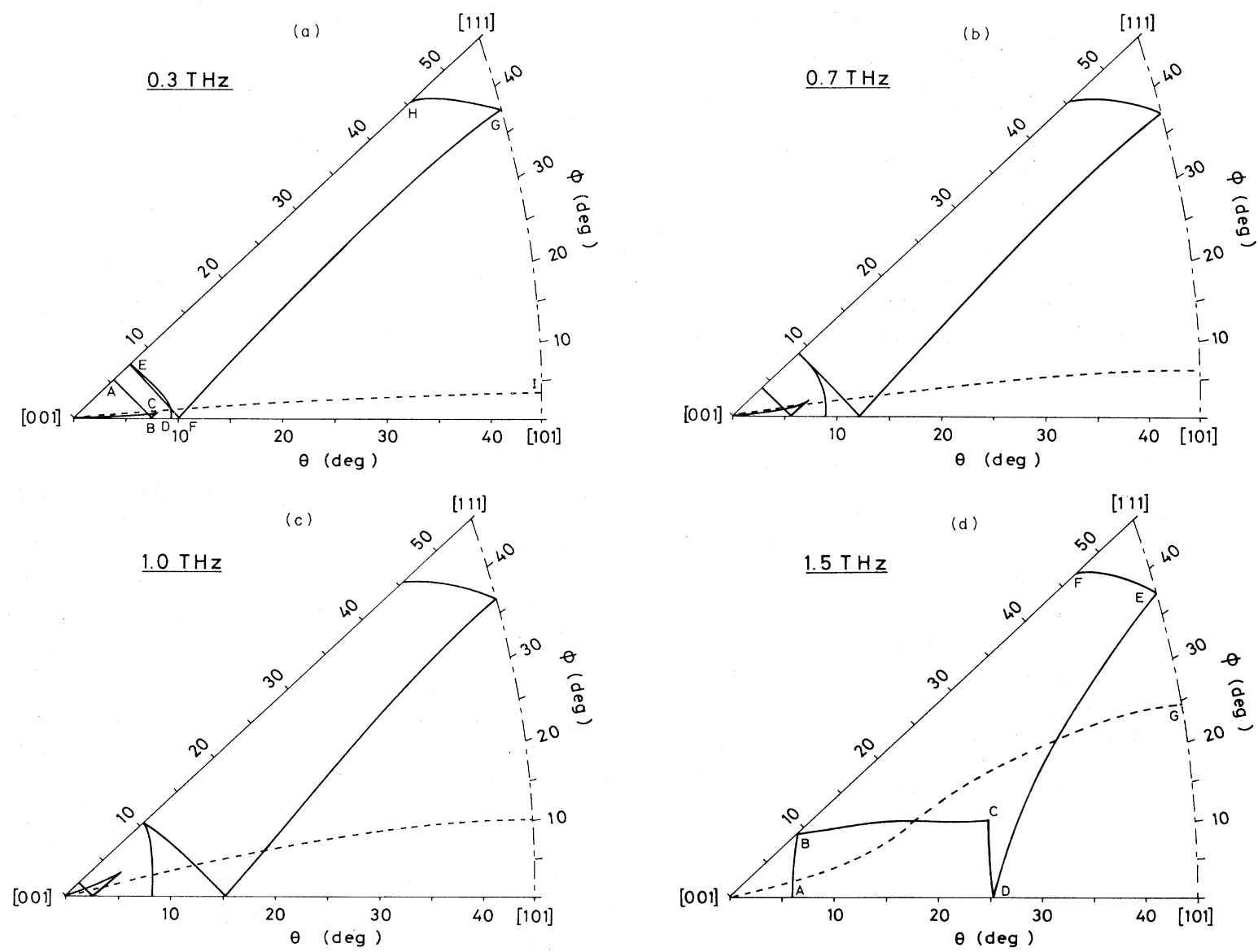

FIG. 5. Polar plots for the sections of caustic surfaces by the fundamental $\frac{1}{48}$ th sector of a unit sphere in the real space. Frequencies selected are (a) $0.3 \mathrm{THz}$, (b) $0.7 \mathrm{THz}$, (c) $1.0 \mathrm{THz}$, and (d) $1.5 \mathrm{THz}$. Solid lines indicate the $\mathrm{T} 1$ mode and dashed lines represent the T2 mode. 


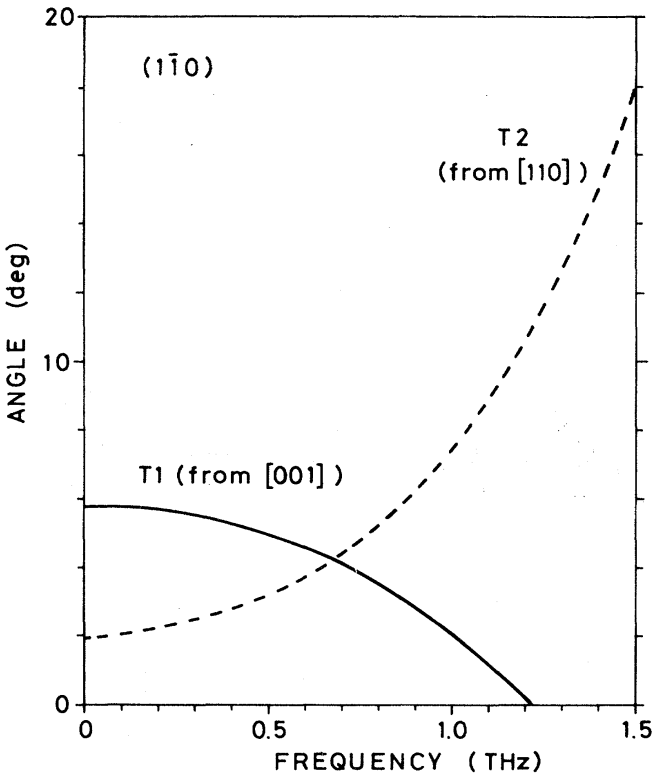

FIG. 6. Calculated locations of the caustic directions vs frequencies in the ( $1 \overline{1} 0)$ plane. Solid line represents the locus of the inner structure of the $\mathrm{T} 1$ caustics in the proximity of the [001] direction. The angle shows the value measured from the [001] direction. Dashed line represents the locus of the T2 caustics measured from the [110] direction.

the concave regions on the $\omega$ surface.) At the same time, the caustics situated outside those mentioned above can also be seen to deform considerably. The departure from the (110) planes of the caustics which extend from the [100] toward [111] directions is remarkable.

It should be noted that the caustics which give rise to a threefold-symmetric cusp structure around the [111] directions remain to exist almost unchanged in all these figures. The presence of this structure at higher frequencies has made the interpretation of the image of the phonons higher than $0.7 \mathrm{THz}$ obtained by the $\mathrm{Pb}$-oxide- $\mathrm{Pb}$ tunneling junction detector somewhat difficult. This is because in the image the focusing structures of the T1 phonons, which develop from the [100] to [111] directions, are missing, including the cusp structures, though they can be seen vividly in the low-frequency image. ${ }^{3}$ Then we have examined more closely the origins of these structures by referring to the geometrical structure of the $\omega$ surfaces. For illustration, the details of the lines of parabolic points on the $\omega$ surfaces at 0.3 and $1.5 \mathrm{THz}$ are displayed in Figs. $7(a)$ and $7(b)$, respectively. Also indicated in these figures by dotted lines are the directions of the wave vectors which lie out of the (110) planes but are accompanied by the group-velocity vector of the $\mathrm{T} 1$ phonons oriented parallel to the same plane. Note that the various points marked $A, B, \ldots$, are mapped onto $A, B, \ldots$, respectively, in Figs. 5(a) and 5(b). It can be shown that the cusps on the caustic lines arise when the direction of vanishing Gaussian curvature touches the parabolic line on the $\omega$ surface. ${ }^{31}$ The points on the $\omega$ surface which yield the cuspidal edges on the caustics are $C$ and $H$ in Fig. 7(a), and $C$ and $F$ in Fig. 7(b). ${ }^{32}$

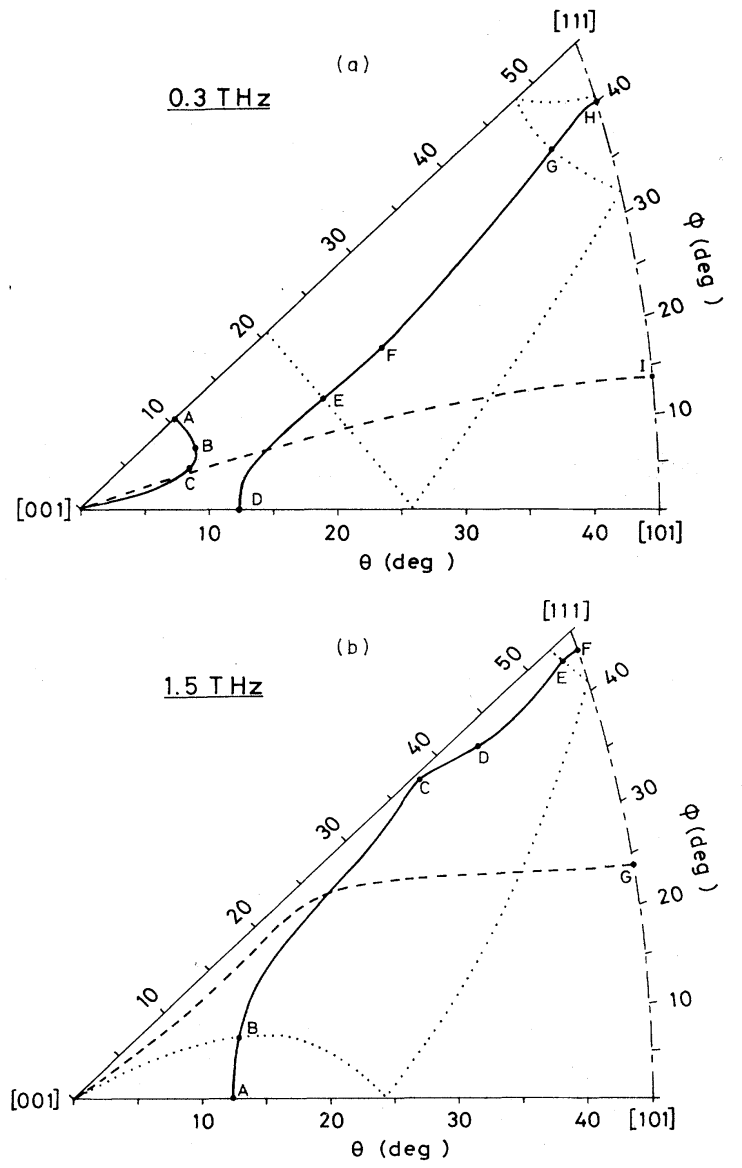

FIG. 7. Parabolic lines on the $\omega$ surfaces at (a) 0.3 and (b) 1.5 THz. Solid lines indicate T1 mode and dashed lines represent the T2 mode. Dotted lines show the directions out of the (110) plane of the wave vectors whose corresponding group-velocity vectors of $\mathrm{T} 1$ phonons point the directions in the (110) plane in the real space. The positions marked by $A, B, \ldots$, are mapped onto $A, B, \ldots$, respectively, in the real space shown in Figs. 5(a) and $5(\mathrm{~d})$.

From Fig. 7(b) we know that at $1.5 \mathrm{THz}$ the points on the comparatively short portion of the parabolic lines between $E$ and $F$, which extend over $\Delta \theta_{\vec{q}}, \Delta \phi_{\vec{q}}=1.5^{\circ}, 0.3^{\circ}$ on the $\omega$ surface, are mapped onto the corresponding long section $E$ to $F$ along the caustics which extend over $\Delta \theta_{\vec{v}}, \Delta \phi_{\vec{v}}=6.5^{\circ}, 8^{\circ}$ in the real space. This suggests that the phonon intensity along the caustics $E$ to $F$ should be reduced considerably as compared with that along the caustics $G$ to $H$ at $0.3 \mathrm{THz}$.

\section{B. Phonon-enhancement maps}

More explicit information on the accumulation of the phonons on the caustics may be gained by plotting the directions of the group-velocity vectors [which are derivable from Eq. (6)] onto the polar plane of the real space. In Figs. $8(\mathrm{a})-8(\mathrm{~d})$, the distributions of the TA mode phonons in the real space can be viewed. In these plots we immediately recognize the presence of heavy accumulation 

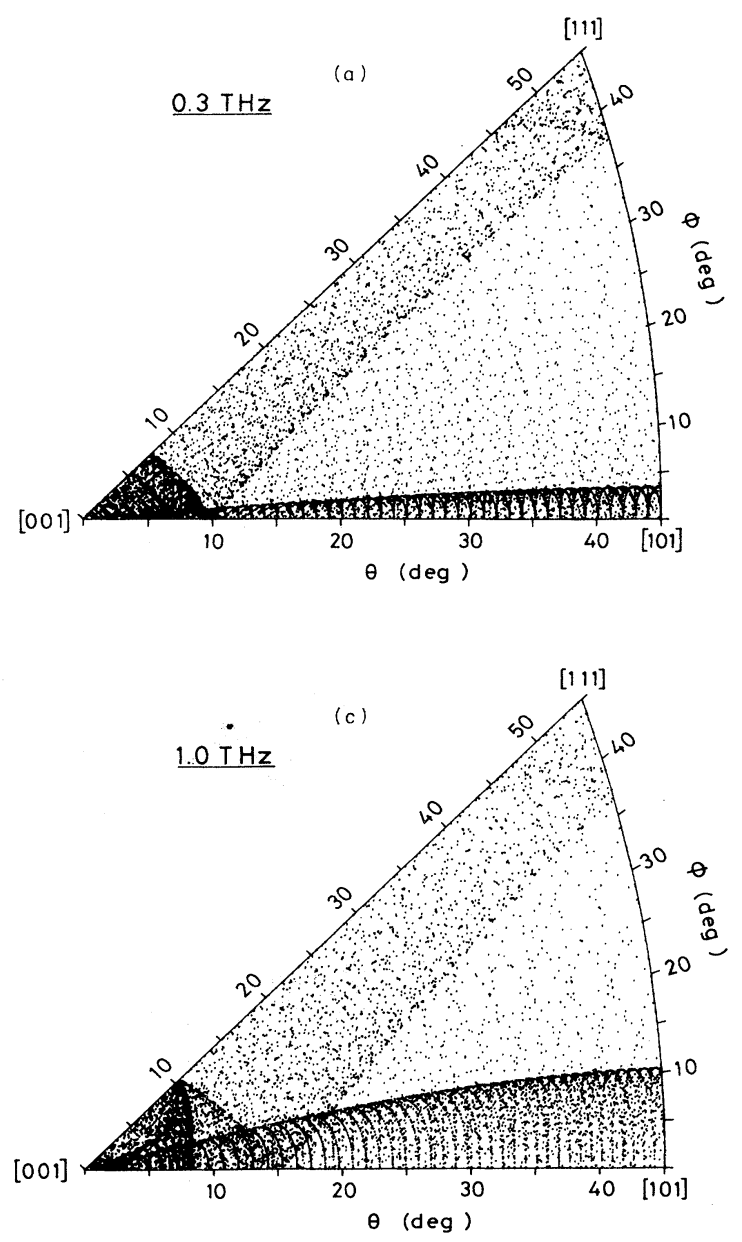
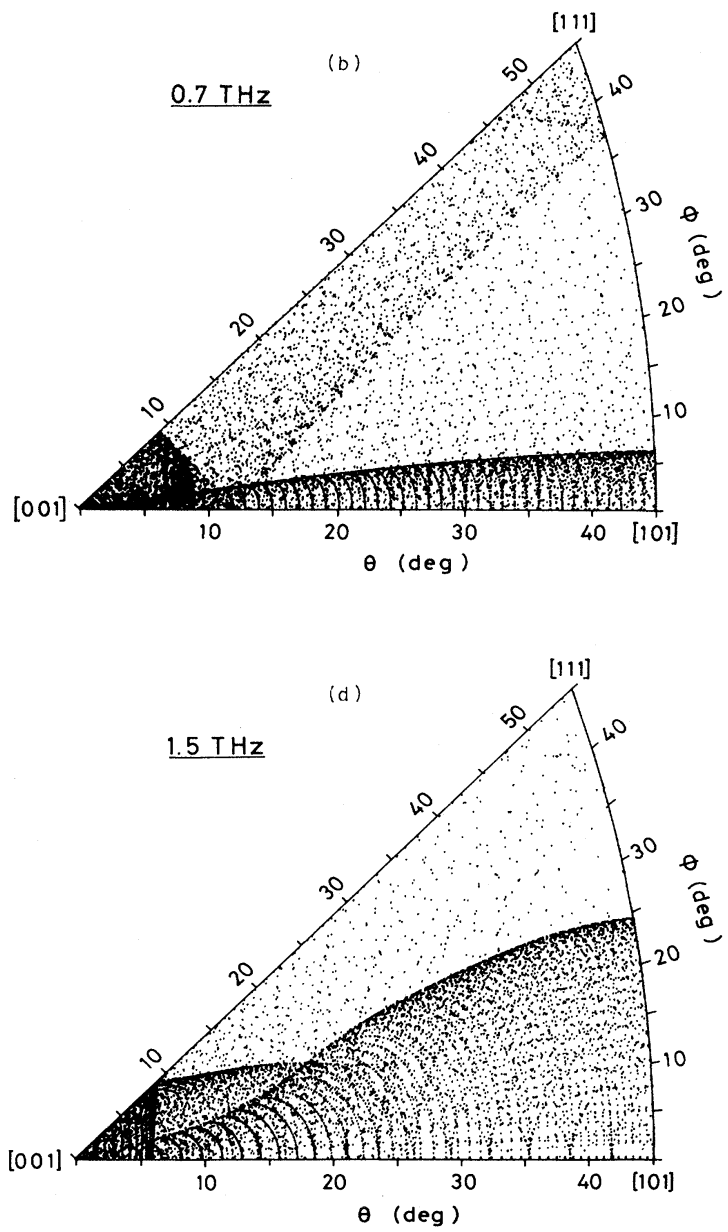

FIG. 8. Phonon distributions in the real space at (a) 0.3 , (b) 0.7 , (c) 1.0 , and (d) $1.5 \mathrm{THz}$. These maps are constructed by plotting the directions of group-velocity vectors which are associated with the wave vectors on the $\omega$ surface distributed with a separation $\delta \theta_{\vec{q}}, \delta \phi_{\vec{q}}=1^{\circ}, 1^{\circ}$ in the sector $0^{\circ} \leq \theta_{\vec{q}} \leq 90^{\circ}$ and $0^{\circ} \leq \phi_{\vec{q}} \leq 45^{\circ}$.

lines of directional points which definitely separate the regions where the phonons are focused from those that are defocused. While we can convince ourselves that the locations of those accumulation lines coincide with those of the caustics shown in Figs. 5(a)-5(d), it is still seen that the caustics do not necessarily associate high concentrations of the points that are discernible from their surroundings. Specifically, the deconcentration of the points on the caustics near the [111] directions is notable at higher frequencies. In fact, a distinctive concentration of the directional points can no longer be seen in the map of 1.5 THz. These results are really in accordance with our expectation stated at the end of Sec. III A.

In the ray picture for the phonons, the phonon flux tends to be infinite on the caustics by Eq. (4). However, the above consideration tells us that the infinity of the phonon flux on the caustics is integrable; that is, when integrated over a finite solid angle (which may correspond to the detection of the flux by a finite-size detector), the phonon flux becomes finite and is not uniform on the caustics.
We also remark that with the development of the structures of the $\mathrm{T} 1$ and $\mathrm{T} 2$ phonons which extend on either side of the (110) and (100) planes, respectively, the density of points within these structures becomes gentle at higher frequencies. All these results explain partly the alteration of the phonon image obtained by the $\mathrm{Pb}$-oxide- $\mathrm{Pb}$ tunneling junction detector from that observed by the Al bolometer which responds rather effectively to the lowerfrequency phonons. ${ }^{3}$ Much more quantitative discussions on the ballistic TA phonon intensities will be given in the next section.

To conclude this section, the caustics and associated TA phonon distributions are expected to change drastically in the $\mathrm{THz}$ frequency region due to acoustic dispersion. An important observation is the fact that the caustics actually play critical roles in the analysis of the phonon focusing, but the search for their locations does not invariably lead to the correct understanding of the behavior of the phonon flux. More elaborate theoretical studies on the phonon distribution or the phonon intensity are required. 


\section{PHONON INTENSITY}

\section{A. Energy flux of the phonons}

Clearly, a quantity which may be obtained directly by experimental measurements is the phonon intensity, or the energy flux of the phonons. This implies that the study on the phonon intensity should provide more direct information for the dispersive effects on the phonon focusing to be tested by the experiments.

Here let us consider the phonons with frequencies in the range $\omega \sim \omega+d \omega$. Then the energy flux $I$ of those phonons of mode $j$, which may be emitted into an infinitesimal solid angle $d \Omega(\overrightarrow{\mathrm{n}})$ around the unit vector $\overrightarrow{\mathrm{n}}$ $(|\overrightarrow{\mathbf{n}}|=1)$ in the real space, is given by

$I(\omega, j ; \overrightarrow{\mathrm{n}}) d \Omega(\overrightarrow{\mathrm{n}}) d \omega$

$$
=\hbar \omega \sum_{\substack{\left\{d^{3} \vec{q}\right\} \\ \overrightarrow{\mathrm{v}}(\overrightarrow{\mathrm{q}}, j) \| \overrightarrow{\mathrm{n}}}} N\left(\overrightarrow{\mathrm{q}}, j ; d^{3} \overrightarrow{\mathrm{q}}\right) v(\overrightarrow{\mathrm{q}}, j) \delta_{\omega, \omega(\overrightarrow{\mathrm{q}}, j)},
$$

where the summation should be taken over possible volume element $d^{3} \overrightarrow{\mathrm{q}}$ in the wave-vector space of the phonons for which the group-velocity vector may be parallel to $\overrightarrow{\mathrm{n}}$, or

$$
\frac{\overrightarrow{\mathrm{v}}(\overrightarrow{\mathrm{q}}, j)}{v(\overrightarrow{\mathrm{q}}, j)}=\overrightarrow{\mathrm{n}}
$$

and $N$ represents the number of the phonons which take part in this flux. This number can be written as

$$
N\left(\overrightarrow{\mathrm{q}}, j ; d^{3} \overrightarrow{\mathrm{q}}\right) \delta_{\omega, \omega(\overrightarrow{\mathrm{q}}, j)}=f(\omega, T) d D_{\omega}(\overrightarrow{\mathrm{q}}, j) d \omega,
$$

where $f$ is a distribution function of the phonons which is assumed here to depend on both the frequency and a temperature $T$ of the local source of the phonons. The explicit expression of $f$, however, relies deeply upon the details of the excitation mechanisms of nonequilibrium phonons though it may be replaced essentially by the Planck distribution at low excitation levels of the phonons. ${ }^{33}$ On the other hand, assuming that the excited phonons are distributed uniformly in the wave-vector space of the crystal, the local phonon density of states $d D_{\omega}$ takes the following form:

$$
d D_{\omega}(\overrightarrow{\mathrm{q}}, j) d \omega=\frac{V}{(2 \pi)^{3}} d S_{\omega}(\overrightarrow{\mathrm{q}}, j) d q_{\perp},
$$

where $V$ is the volume of the excitation region, $d S_{\omega}$ is an element of area on the surface of the selected constant frequency $\omega=\omega(\vec{q}, j)$ in the wave-vector space, and $d q_{\perp}$ denotes the perpendicular distance between the surfaces $\omega$ constant and $\omega+d \omega$ constant, i.e.,

$$
d q_{\perp}=\frac{d \omega(\overrightarrow{\mathrm{q}}, j)}{v(\overrightarrow{\mathrm{q}}, j)} .
$$

Accordingly, it is derived that

$$
I(\omega, j ; \overrightarrow{\mathrm{n}})=\frac{V}{(2 \pi)^{3}} \hbar \omega f(\omega, T) \sum \frac{d S_{\omega}(\overrightarrow{\mathrm{q}}, j)}{d \Omega(\overrightarrow{\mathrm{n}})}
$$

where the sum is taken over possible surface elements for which the surface normal becomes collinear with the vector $\overrightarrow{\mathrm{n}}$. Introducing further the phase velocity $c$ and making use of the angle $\theta$ between $\vec{q}$ and $\vec{v}$, Eq. (14) may also be written as

$$
I(\omega, j ; \overrightarrow{\mathrm{n}})=\frac{V}{(2 \pi)^{3}} \hbar \omega^{3} f(\omega, T) \int \frac{\delta_{\omega, \omega(\overrightarrow{\mathrm{q}}, j)}}{c^{2}(\overrightarrow{\mathrm{q}}, j) \cos \theta(\overrightarrow{\mathrm{q}}, j)} \frac{d \Omega(\overrightarrow{\mathrm{q}}, j)}{d \Omega(\overrightarrow{\mathrm{n}})} \delta^{2}\left[\overrightarrow{\mathrm{n}}-\frac{\overrightarrow{\mathrm{v}}(\overrightarrow{\mathrm{q}}, j)}{v(\overrightarrow{\mathrm{q}}, j)}\right] d \Omega(\overrightarrow{\mathrm{q}}, j)
$$

with the definition

$$
\int \delta^{2}\left[\overrightarrow{\mathrm{n}}-\frac{\overrightarrow{\mathrm{v}}(\overrightarrow{\mathrm{q}}, j)}{v(\overrightarrow{\mathrm{q}}, j)}\right] d \Omega(\overrightarrow{\mathrm{q}}, j)=1 .
$$

Hence identifying $d \Omega(\overrightarrow{\mathrm{n}})$ with $d \Omega(\overrightarrow{\mathrm{v}}(\overrightarrow{\mathrm{q}}, j))$, we have

$$
I(\omega, j ; \overrightarrow{\mathrm{n}})=\frac{V}{(2 \pi)^{3}} \hbar \omega^{3} f(\omega, T) W(\omega, j ; \overrightarrow{\mathrm{n}}),
$$

where

$$
\begin{aligned}
W(\omega, j ; \overrightarrow{\mathrm{n}})=\int & \frac{\delta_{\omega, \omega(\overrightarrow{\mathrm{q}}, j)}}{c^{2}(\overrightarrow{\mathrm{q}}, j) \cos \theta(\overrightarrow{\mathrm{q}}, j)} A(\overrightarrow{\mathrm{q}}, j) \\
& \times \delta^{2}\left[\overrightarrow{\mathrm{n}}-\frac{\overrightarrow{\mathrm{v}}(\overrightarrow{\mathrm{q}}, j)}{v(\overrightarrow{\mathrm{q}}, j)}\right) d \Omega(\overrightarrow{\mathrm{q}}, j) .
\end{aligned}
$$

Thus it can be seen that the enhancement factor $A$ of the phonon flux plays the central role in determining the phonon intensity. Incidentally, $\cos \theta(\overrightarrow{\mathrm{q}}, j)=c(\overrightarrow{\mathrm{q}}, j) / v(\overrightarrow{\mathrm{q}}, j)$ holds for nondispersive phonons, and in this case Eq. (15) is similar to the expression derived by Rösch and Weis for the phonon flux in thermal equilibrium. ${ }^{34}$

Now plotted in Figs. 9(a) and 9(b) are the angular distributions of $W$ 's and their sum in the (110) plane of the TA phonons at frequencies 0.3 and $1.5 \mathrm{THz}$, respectively. Note that the angular dependence of the phonon intensity is exclusively included in $W$, and its sharp features stem essentially from the phonon-enhancement factor $A$. This is because the factor $c^{2} \cos \theta$ in Eq. (18) depends rather moderately upon the direction and acts only to reduce the phonon intensity of the $\mathrm{T} 2$ mode relative to the $\mathrm{T} 1$ mode, and also to reduce the overall magnitude of $W$ at lower frequencies.

Taking these factors into account, the structures at 0.3 $\mathrm{THz}$ are found to be nearly identical to that of the phonon intensity in the low-frequency limit. ${ }^{20,29}$ Comparing with the results at $0.3 \mathrm{THz}$, the reduction of the phonon intensity in the neighborhood of the [111] direction is indeed remarkable in the figure at $1.5 \mathrm{THz}$. The directions marked by arrows in these figures are those at which the caustics intersect with the (110) plane [see Figs. 5(a) and 5(d)]. The positions of the arrows coincide well with the directions along which the phonon intensity is amplified 

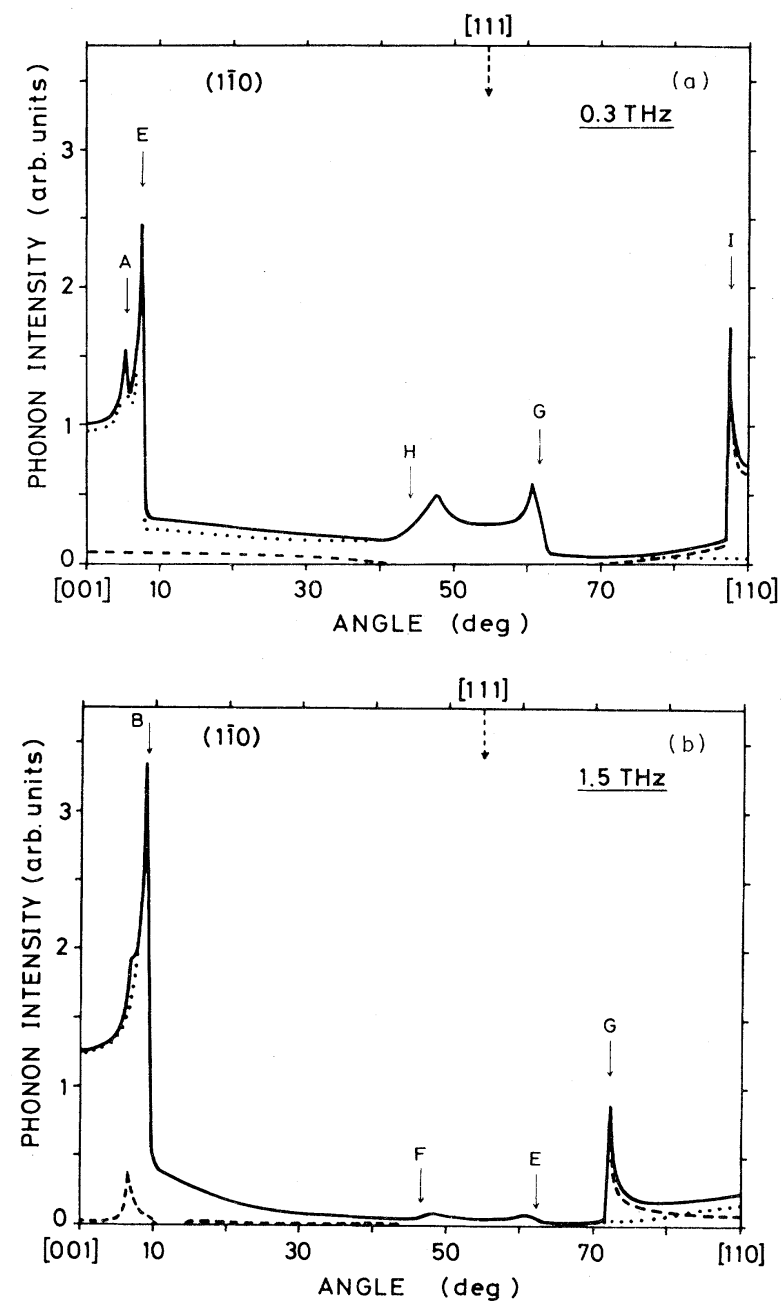

FIG. 9. Angular dependences of TA phonon intensities in the (110) plane integrated over $0.25^{\circ}$ and $2.5^{\circ}$ within and perpendicular to the plane, respectively. Frequencies are (a) 0.3 and (b) 1.5 THz. Dotted and dashed lines represent the contributions of $\mathrm{T} 1$ and T2 phonons, respectively. Solid lines are their sum. Positions indicated by arrows show the directions at which the caustic surfaces intersect with the (110) plane [cf. Figs. 5(a) and $5(\mathrm{~d})]$.

strongly. Slight deviations of $H$ and $G$ at $0.3 \mathrm{THz}$ and of $F$ and $E$ at $1.5 \mathrm{THz}$ from the peak locations are the effects of finite magnitude of $d \Omega(\overrightarrow{\mathrm{n}})$ assumed in the calculations. When we postulate a finer angular resolution of the phonon detection perpendicular to the ( $1 \overline{1} 0)$ plane, more satisfactory coincidences are obtained. Another characteristic feature observed in the phonon intensity at $1.5 \mathrm{THz}$ is the present of the sharp hump in the T2 mode which is located at $6.5^{\circ}$ measured from the [001] axis. The origin of this

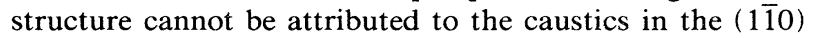
plane and will be discussed below.

\section{B. Group-velocity surfaces}

Further insight into the origins of various singular behaviors of the phonon flux including the one mentioned above may be provided by considering the group-velocity surfaces. In Fig. 10 the (110) sections of the groupvelocity surfaces of the TA phonons are plotted for 0.3 and $1.5 \mathrm{THz}$. This figure is obtained by selecting a set of group-velocity vectors which lie within $\pm 0.1^{\circ}$ of the (110) plane. Hence the density of the plotted points represents again the concentration of the phonons in the real space.

Apparently these surfaces are considerably complex in comparison with the $\omega$ surfaces in the wave-vector space. It is, on one hand, due to the fact that the transitions of the phonon modes from $\mathrm{T} 1$ to $\mathrm{T} 2$ and vice versa happen. At lower frequencies these transitions turn up, owing to the intersection of the $\omega$ surfaces of two TA modes in the [111] direction. At higher frequencies, e.g., $1.5 \mathrm{THz}$, additional intersections take place due to large deformations of the $\omega$ surfaces. At $0.3 \mathrm{THz}$ the directions along which the $\mathrm{T} 2$ phonons cannot propagate in the $(1 \overline{1} 0)$ plane are those within $\pm 14.3^{\circ}$ on either side of the [111] axis, whereas at $1.5 \mathrm{THz}$ they become $10.0^{\circ}$ to $15.0^{\circ}$ rotated away from the [001] axis, in addition to those within $\pm 10.6^{\circ}$ on either side of the [111] axis.

The more substantial complexity stems from the multivaluedness of the surfaces, reflecting the fact that the phonon flux of a TA mode in a given direction consists generally of more than one phonon traveling with group velocities different in magnitude. (They should, in principle, be observed separately by the high-resolution time-offlight experiments.) In particular, certain phonons in the T1 branch whose wave vectors lie outside the (1T) plane can still have group velocities in this plane. The locations of the corresponding wave vectors on the $\omega$ surfaces have already been depicted in Figs. 7(a) and 7(b). The groupvelocity surfaces which are constructed by these phonons are those branches in which no transition points to the T2 phonons exist in Fig. 10.

Now the comparison of these traces at different frequencies enables us to identify some characteristic changes of the group-velocity surface other than the overall shrinkage at the higher frequencies (which is evidently due to the dispersion). One of the observations may be the remarkable growth at the higher frequency of the sectioned fold of the T2 mode in the vicinity of the [110] direction, which results from the development of the negative curvature region of the $\omega$ surface near the [110] direction [see Fig. 3(b)]. It has been well established that the sectioned fold edges of the group-velocity surfaces are originated from the parabolic points on the associated $\omega$ surfaces and thereupon indicate the caustic directions. The positions of the caustics in the (110) plane shown in Figs. 5(a) and 5(b) are really in one-to-one correspondence with the fold edges marked in Fig. 10. Here we remark that at the points $H$ of $0.3 \mathrm{THz}$ and $F$ of $1.5 \mathrm{THz}$, the complete surface terminates making a cuspidal edge, though it cannot be seen so obviously due to an overlap with the surface of another branch.

Another outstanding feature observed in Fig. 10 may be the presence of a sharp extremum (equal to $2.85 \times 10^{5}$ $\mathrm{cm} / \mathrm{s}$ ) in the trace of $1.5 \mathrm{THz}$ situating at $7.0^{\circ}$ measured from the [001] direction. The appearance of this local extremum of the group velocity stems from the fact that for the T2 mode, the contour lines of constant frequency in the wave-vector space [Fig. 3(b)] are in close order around the corresponding direction, i.e., about $25.5^{\circ}$ to the [001] 


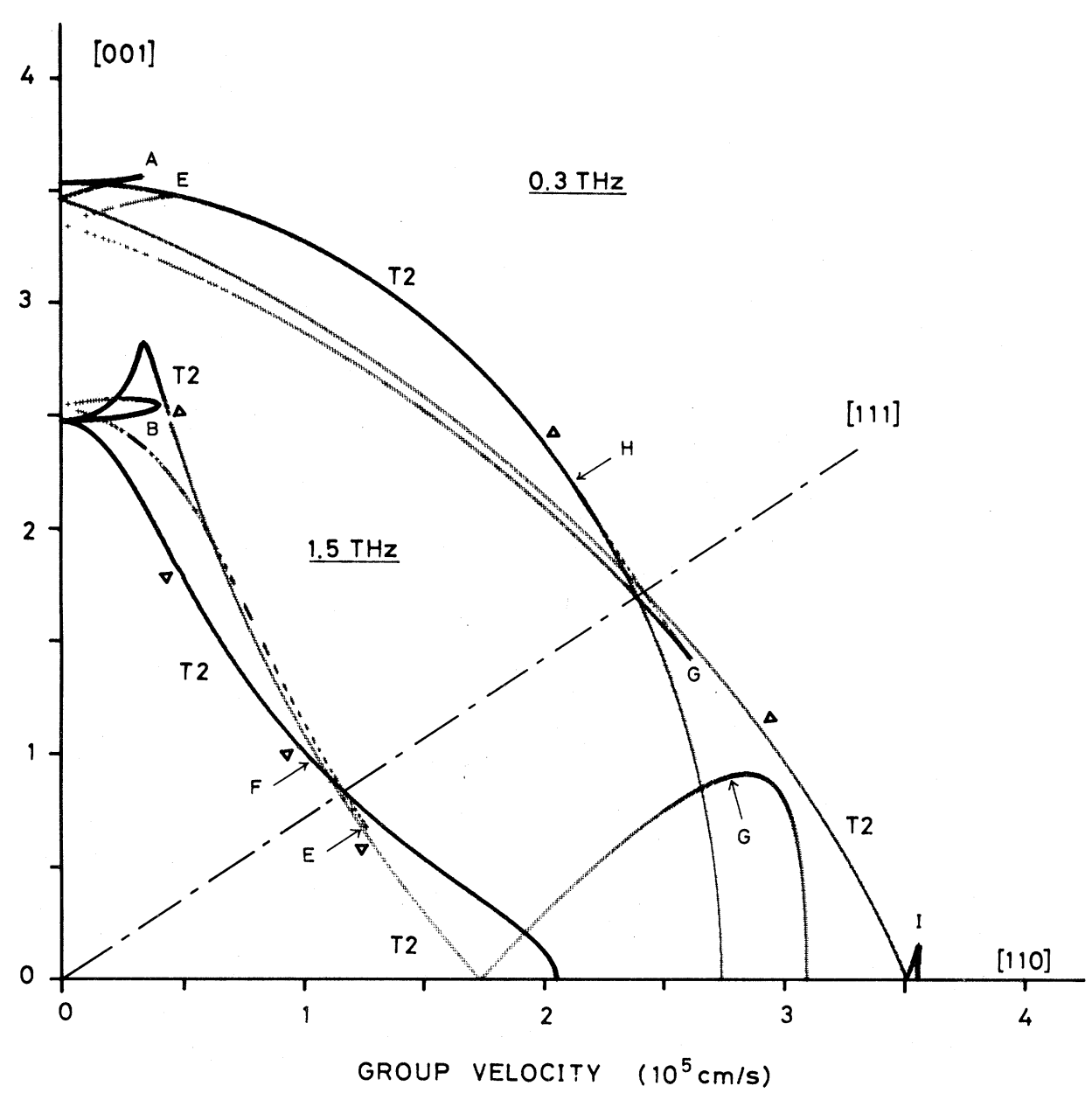

FIG. 10. Sections by the (110) plane of group-velocity surfaces of TA phonons at 0.3 and $1.5 \mathrm{THz}$. Wave vectors on each $\omega$ surface distributed with a separation $\delta \theta_{\vec{a}}, \delta \phi_{\vec{q}}=0.1^{\circ}, 0.1^{\circ}$ have been used to generate a set of group-velocity vectors. Each point represents one of these vectors which lie within $0.1^{\circ}$ of the (110) plane. The open triangles indicate the positions at which the transitions from $\mathrm{T} 1$ to $\mathrm{T} 2$ and vice versa happen. These surfaces fold at points marked by $A, B, \ldots$, and their directions coincide with the locations of the corresponding points $A, B, \ldots$, in Figs. 5(a) and 5(d).

axis as well as around the [110] direction. Incidentally, note that at the same frequency the sectioned $\omega$ surface of the $\mathrm{T} 2$ phonons possesses a portion which is regarded to be approximately flat over a finite range of directions, e.g., $19^{\circ}$ to $23^{\circ}$ rotated away from the [001] axis. The existence of this portion of the $\omega$ surface gives rise to the strong enhancement of the phonon intensity to be observed in the T2 phonons near the [001] direction, which cannot be attributed to the caustics. However, at frequencies higher than $1.5 \mathrm{THz}$, this hump of the T2 phonons will be replaced by the sharp ridges of the phonon intensity characteristic to the caustics because the curvature of the corresponding sections of the $\omega$ surfaces changes its sign at a frequency near $1.5 \mathrm{THz}$.

\section{ISOTOPE EFFECTS}

The effects of the phonon-isotope interaction upon the ballistic transmission of near $1-\mathrm{THz}$ phonons are now discussed. Owing to the presence of the scattering by the iso- topes, the ballistic component of the phonon intensity in $\mathrm{Ge}$ is attenuated. The lifetime of the phonons limited by the isotope scattering is described by the relaxation time which is spatially isotropic and independent of the polarizations of the phonons, i.e., ${ }^{15}$

$$
\tau^{-1}(\omega)=\frac{\pi}{6} V_{0} g \omega^{2} \mathscr{D}(\omega),
$$

where $V_{0}$ is the volume per atom and $\mathscr{D}$ denotes the onephonon density of states shown in Fig. 2. The constant $g$ is defined by

$$
g=\sum_{i} \gamma_{i}\left(1-m_{i} / \bar{m}\right)^{2},
$$

where $\gamma_{i}$ and $m_{i}$ are the relative fraction and mass of the $i$ th isotope, and $\bar{m}$ is the average mass of all atoms. For $\mathrm{Ge}, \mathrm{g}$ takes a value of $5.87 \times 10^{-4}$. In the low-frequency limit, $\mathscr{D}(\omega) \propto \omega^{2}$ and then $\tau^{-1} \propto \omega^{4}$. However, near 1THz frequencies $\mathscr{D}(\omega)$ grow more rapidly than $\omega^{2}$ (see Fig. 2). The effects of the dispersion upon $\tau^{-1}$ amount to 
about $20 \%$ at $1 \mathrm{THz}$ and become severe at zone-boundary frequencies.

In the presence of the isotopes, the expression for the phonon intensity (18) should be modified as

$$
A(\overrightarrow{\mathrm{q}}, j) \rightarrow A(\overrightarrow{\mathrm{q}}, j) \exp [-t(\overrightarrow{\mathrm{q}}, j) / \tau(\omega)],
$$

where $t(\overrightarrow{\mathrm{q}}, j)=d / v(\overrightarrow{\mathrm{q}}, j)$ with $\overrightarrow{\mathrm{v}}(\overrightarrow{\mathrm{q}}, j) \| \overrightarrow{\mathbf{n}}$ is the ballistic time of flight of the relevant phonons that traverse the distance $d$ between the phonon source and the detector.

We have displayed in Fig. 11 the transmission rate of the ballistic phonons which travel along three principal directions of the cubic crystals and then arrive at the detector without being subject to the scattering. The configurations of the phonon detection assumed in the evaluation of $t$ are just those employed in the experiments by Dietsche $e t a l^{3}$; that is, the phonon source is assumed to be scanned across a (1T0) surface of a Ge crystal and the arrival of the phonons is observed with a fixed detector on the opposite face at a distance of $0.5 \mathrm{~mm}$. As we have pointed out repeatedly, the TA phonons which can propagate in one direction consist, in general, of several branches. The results shown in this figure are those of the phonons for which $\vec{q}$ becomes collinear with $\vec{v}$ for the [100] and [110] propagations (i.e., pure mode phonons), and $\vec{q}$ lies in the (1) 10 ) plane for the [111] propagation. For the propagation in the [110] direction, the only results of the T2 phonons which focus strongly in this direction are represented.

Now the ballistic components of the phonons can be seen to decrease very rapidly at $v>0.3 \mathrm{THz}$. At the

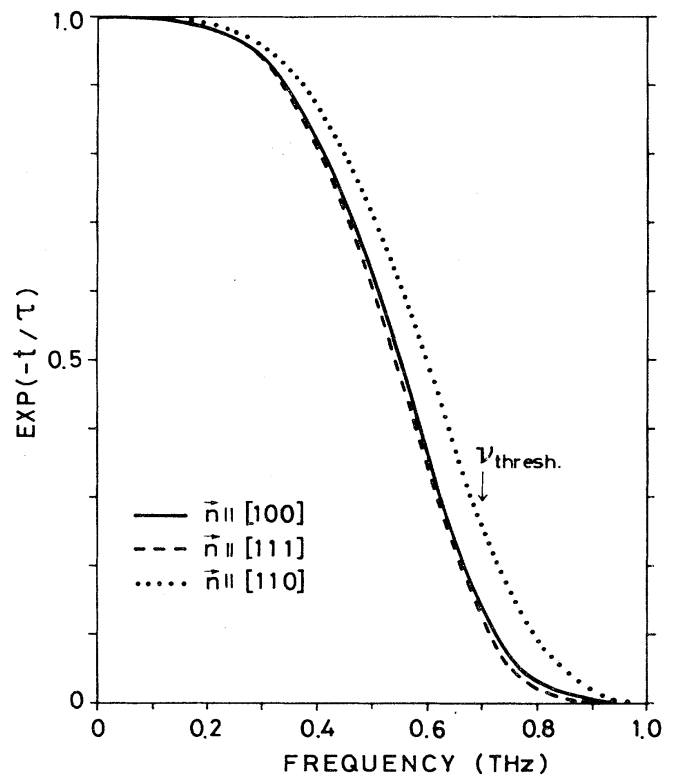

FIG. 11. Theoretical rates of ballistically transmitted phonons under the presence of the isotope scattering in the experimental configuration by Dietsche et al. (Ref. 3); solid line, [100] propagation $(\vec{q} \|[100]$, both $\mathrm{T} 1$ and $\mathrm{T} 2)$ with a path length $0.5(\sqrt{2}) \mathrm{mm}$; dashed line, [111] propagation [with $\overrightarrow{\mathrm{q}}$ parallel to the (110) plane, T1] with path length $0.5(\sqrt{3 / 2}) \mathrm{mm}$; dotted line, [110] propagation $(\overrightarrow{\mathrm{q}} \|[110], \mathrm{T} 2)$ with a path length $0.5 \mathrm{~mm}$. $v_{\text {thresh }}=0.7 \mathrm{THz}$ is the lowest frequency for which the $\mathrm{Pb}$-oxide$\mathrm{Pb}$ tunneling junction detector can respond. threshold frequency, i.e., $0.7 \mathrm{THz}$, of the $\mathrm{Pb}$-oxide- $\mathrm{Pb}$ tunneling junction detector, the rate of the unscattered phonons which may be detected is $26 \%$ for the [110] propagation but it amounts to $14 \%$ and $12.5 \%$ for the [100] and [111] propagations, respectively. The scattering of the phonons traveling in the [111] direction is more frequent than those in the [100] and [110] directions, owing to the smallness of the group velocity, e.g., $v_{[111]}=2.76 \times 10^{5}$, $v_{[100]}=3.36 \times 10^{5}$, and $v_{[110]}=3.48 \times 10^{5}$ in units of $\mathrm{cm} \mathrm{s}^{-1}$, and also owing to a relatively long path length of $d=0.5(\sqrt{3 / 2}) \mathrm{mm}$ for this propagation of the assumed configuration (incidentally, $d=0.5(\sqrt{2}) \mathrm{mm}$ for the [100] propagation). Accordingly, in the experimental arrangements by Dietsche et al., ${ }^{3}$ the scattering of the phonons by naturally occurring isotopes in Ge results in significantly reducing the ballistic phonon intensities which are observed in the [100] and [111] directions. As remarked in Sec. III, the experimental value of an opening angle of the $\mathrm{T} 2$ phonon caustics obtained by the tunneling junction detector corresponds to the phonon frequency of $0.8 \mathrm{THz}$. At this frequency, the isotope scattering of the phonons renders the rate of the ballistic transmission of the T2 phonon intensity to be $9 \%$ in the [110] direction. These observations convince us that the detection of quasimonochromatic phonons in a narrow band of frequencies $0.7-0.8 \mathrm{THz}$ is really intelligible.

We further comment on the absence of the threefold cusp structures of the focusing in the image of the phonons higher than $0.7 \mathrm{THz}$. This has partly been explained before by the dispersive effects on the phonon focusing. However, as we can understand from Fig. 8, it may be rather hard to account for quantitatively the reason based only on the dispersion. This is because the theoretical phonon distribution pattern near the cusp of $0.7-\mathrm{THz}$ phonons is not so drastically modified from the corresponding pattern of $0.3-\mathrm{THz}$ phonons which may be observed by the Al bolometer. In this connection the scattering of the phonons by isotopic disorder in Ge should again play an important role. Indeed, due to the presence of this scattering mechanism, it is only $12.5 \%$ of $0.7-\mathrm{THz}$ phonons emanated in the [111] direction that can respond to the detector as unscattered ballistic phonons, whereas they amount to $94 \%$ at $0.3 \mathrm{THz}$. A more complete analysis of the phonon intensity, including the effects of the phonon scattering, requires further knowledge on the distribution function $f(\omega, T)$ together with the value of $T$ which describes the local temperature of the phonon source.

To conclude, in order to verify experimentally the predicted focusing structure near the [111] direction, much thinner samples, or the configurations in which the path length of the phonons for the [111] propagation becomes much shorter, should be prepared.

\section{CONCLUDING REMARKS}

In this paper we have given a detailed theoretical analysis for the focusing of the TA phonons in Ge with frequencies ranging $0.3-1.5 \mathrm{THz}$. Our calculations are based on a lattice-dynamical model which very accurately describes the phonon dispersion relations. A complementary study of various quantities which account for the directional characteristics of the phonons, such as the caustics, phonon distributions in the real space, and angu- 
lar dependence of the phonon intensity, reveals considerable alterations of the focusing properties of the phonons at frequencies higher than $0.3 \mathrm{THz}$. If we appropriately take into consideration the isotope effects upon phonon conduction which become significant at frequencies near the $1-\mathrm{THz}$ range, our results are in good accordance with the experimental findings by Dietsche et al., ${ }^{3}$ including the absence of the structure near the [111] directions.

The results exhibited extensively in the present work are those on Ge. However, the same kinds of behaviors of the phonon focusing at dispersive frequencies are expected as well for $\mathrm{GaAs}$ and $\mathrm{Si}$. This is because their dispersion curves are very similar to those of Ge characterized by marked flattening of the TA branches in the [111] and [100] directions. Especially, for GaAs, even the magnitudes of the frequencies lying on the dispersion curves of acoustic branches are very close to those of $\mathrm{Ge}$, while $\mathrm{Si}$ is much harder than Ge.

In contrast, the isotope effects upon the phonon conduction in GaAs is expected to be much weaker than those in Ge. This relies deeply on the fact that the element As is isotopically pure and that Ga consists of only two isotopic atoms even though $\mathrm{Ge}$ is a mixture of five isotopes. The quantity $g$ for GaAs is $1.97 \times 10^{-4}$, which is nearly onethird of the corresponding value for Ge. Furthermore, it has been shown that the lattice vibrations of As do not contribute to the scattering, and the effective one-phonon density of states available for the final states of the scattered phonons is also reduced to about one-half or less in comparison with that of Ge. ${ }^{35}$ Accordingly, the effects of the isotopes for the phonons in GaAs are estimated to be 1 order of magnitude smaller than those in Ge. This implies that the detection of the ballistic propagation of the dispersive phonons is more feasible in GaAs that in $\mathrm{Ge}$.
${ }^{1}$ R. G. Ulbrich, V. Narayanamurti, and M. A. Chin, Phys. Rev. Lett. 45,1432 (1980).

${ }^{2}$ P. $\mathrm{Hu}$, V. Narayanamurti, and M. A. Chin, Phys. Rev. Lett. 46, 192 (1981).

${ }^{3}$ W. Dietsche, G. A. Northrop, and J. P. Wolfe, Phys. Rev. Lett. 47, 660 (1981).

${ }^{4}$ H. Lengfellner and K. F. Renk, Phys. Rev. Lett. 뜨, 1210 (1981).

${ }^{5}$ R. Baumgartner, M. Engelhardt, and K. F. Renk, Phys. Rev. Lett. 47, 1403 (1981).

${ }^{6}$ K. T. Tsen, D. A. Abramsohn, and R. Bray, Phys. Rev. B $\underline{26}$, 4770 (1982).

${ }^{7}$ D. B. McWhan, P. Hu, M. A. Chin, and V. Narayanamurti, Phys. Rev. B 26, 4774 (1982).

${ }^{8}$ For a review, see W. Bron, Rep. Prog. Phys. 43, 301 (1980).

${ }^{9}$ S. Tamura, Phys. Rev. B 25, 1415 (1982).

${ }^{10}$ G. A. Northrop, Phys. Rev. B 26, 903 (1982).

${ }^{11}$ B. Taylor, H. J. Maris, and C. Elbaum, Phys. Rev. B $\underline{3}, 1462$ (1971).

${ }^{12}$ H. J. Maris, J. Acoust. Soc. Am. 50, 812 (1971).

${ }^{13}$ R. Orbach and L. A. Vredevoe, Physics (N.Y.) 1,91 (1964).

${ }^{14} \mathrm{G}$. Klemens, in Solid State Physics, edited by F. Seitz and D. Turnbull (Academic, New York, 1958), Vol. 7.

15S. Tamura, Phys. Rev. B 27, 858 (1983).

${ }^{16}$ G. Nilsson and G. Nelin, Phys. Rev. B $\underline{3}, 364$ (1971).

${ }^{17}$ G. Nelin and G. Nilsson, Phys. Rev. B 5, 3151 (1972).

${ }^{18}$ M. Born and K. Huang, Dynamical Theory of Crystal Lattices (Oxford, New York, 1954).

${ }^{19}$ P. Taborek and D. L. Goodstein, Solid State Commun. $\underline{33}$,
1191 (1980); Phys. Rev. B 22, 1550 (1980).

20J. C. Hensel and R. C. Dynes, Phys. Rev. Lett. 43, 1033 (1979).

${ }^{21}$ G. A. Northrop and J. P. Wolfe, Phys. Rev. Lett. $\underline{43}, 1424$ (1979).

${ }^{22}$ A. A. Maradudin, E. W. Montroll, G. H. Weiss, and I. P. Ipatova, Theory of Lattice Dynamics in the Harmonic Approximation, 2nd ed. (Academic, New York, 1971).

${ }^{23}$ H. J. McSkimin, J. Appl. Phys. 24, 988 (1953).

${ }^{24}$ F. Herman, J. Phys. Chem. Solids $\underline{8}, 405$ (1959).

${ }^{25}$ A. D. Zdetsis and C. S. Wong, Phys. Rev. B 19,2999 (1979).

${ }^{26}$ The corrections of phonon frequencies due to the isotope effects are very small in Ge. See Ref. 15.

${ }^{27}$ This figure is made by employing a linear analytic method devised by G. Gilat and L. Raubenheimer, Phys. Rev. B 4, 390 (1966).

${ }^{28}$ G. A. Northrop and J. P. Wolfe, Phys. Rev. B 22, 6196 (1980). ${ }^{29}$ S. Tamura and Y. Nakane, Phys. Rev. B 24, 4317 (1981).

${ }^{30}$ M. Lax and V. Narayanamurti, Phys. Rev. B 22, 4876 (1980).

${ }^{31}$ M. V. Berry, Adv. Phys. 25, 1 (1976).

${ }^{32}$ The locations of points on the $\omega$ surface which give rise to cuspidal edges on the caustics have been investigated extensively by Every for various cubic crystals in the low-frequency limit, A. G. Every, Phys. Rev. B 24, 3456 (1981).

${ }^{33}$ W. E. Bron and W. Grill, Phys. Rev. B 16, 5303 (1977).

${ }^{34}$ F. Rösch and O. Weis, Z. Phys. B $\underline{33}, 101$ (1977).

${ }^{35}$ M. Lax, V. Narayanamurti, P. Hu, and W. Weber, J. Phys. (Paris) Colloq. 42, C6-161 (1981). 